\title{
How effective are reedbeds, ponds, restored and constructed wetlands at retaining nitrogen, phosphorus and suspended sediment from agricultural pollution in England?
}

\author{
Elizabeth J Palmer-Felgate ${ }^{* *}$, Mike C Acreman', Jos TA Verhoeven², Miklas Scholz ${ }^{3}$, Edward Maltby ${ }^{4}$, \\ Charlie J Stratford ${ }^{1}$, Jonathan Newman ${ }^{1}$, James Miller ${ }^{1}$ and Deborah Coughlin ${ }^{5}$
}

\begin{abstract}
A high priority topic within the Department for Environment, Food and Rural Affairs (DEFRA) water quality programme is the mitigation of diffuse rural pollution from agriculture. Wetlands are often cited as being effective at reducing nutrient and sediment loadings to receiving waters. However, the research in this area is inconsistent, and whilst most studies have shown that both natural and constructed wetlands retain nutrients and sediments, others have shown that they have little effect, or even increase nutrient and sediment loads to receiving water bodies. DEFRA has commissioned a systematic review on the use of wetlands to mitigate N, P and SS inputs from agriculture to receiving freshwater in England. The review will encompass a comprehensive literature search on all available material on the subject, both published and unpublished within the British Isles. Specific inclusion criteria will be adhered to and a formal assessment of the quality and reliability of the studies will be undertaken. The data will then be extracted and a data synthesis undertaken. The review will inform an evidence-based policy that can be implemented by stakeholders.
\end{abstract}

Keywords: Wetlands, Reedbeds, Ponds, Constructed, Restored, Nutrients, Nitrogen, Phosphorus, Suspended Sediment, Agriculture

\section{Background}

A high priority topic within the Department for Environment, Food and Rural Affairs (DEFRA) water quality programme is the mitigation of diffuse rural pollution from agriculture. This programme was set up to meet the requirements of the European Water Framework Directive (WFD ) [1]. Nutrient loss from agricultural land has been suggested as a major cause of elevated nutrient concentrations in surface waters in the UK [2]. Nitrogen (N) and phosphorus $(\mathrm{P})$ are of particular concern as an excess of either nutrient can lead to eutrophication of freshwater systems and coastal waters. Agriculture has also been identified as a significant source of suspended sediment concentrations in UK rivers [3]. Suspended sediment (SS)

\footnotetext{
* Correspondence: ejsu@ceh.ac.uk

'Centre for Ecology and Hydrology, CEH Wallingford, Maclean Building,

Crowmarsh Gifford, Wallingford, Oxfordshire OX10 8BB, UK

Full list of author information is available at the end of the article
}

can lead to loss of ecological integrity [4] and agriculturally derived sediment has been identified as a source of increased bed-sediment $\mathrm{P}$ concentrations in rivers [5].

Wetlands are often cited as being effective at reducing nutrient and sediment loadings to receiving waters. However, the research in this area is inconsistent, and whilst most studies have shown that both natural and constructed wetlands retain nutrients and sediments, others have shown that they have little effect, or even increase nutrient and sediment loads to receiving water bodies [6-8]. Many factors may have contributed to these disparate results, including the length of time the wetland has been established for, seasonality, the hydrogeomorphic landscape setting, type, size, level and type of management, and the input concentrations/loads and historic loading of the wetland. For example, a constructed wetland system in South-west England switched from a net annual sink to a source of phosphorus over a 
10 year period, and from being a sink to a source of soluble reactive phosphorus (SRP) and ammonium between spring and summer $[9,10]$. Phosphorus removal has been shown to correlate positively with an increase in wetland area, and a minimum wetland to farmyard area ratio of 1.3 has been proposed for effective removal of molybdate reactive phosphorus (MRP) [11]. When deciding on wetland size, other factors such as ecosystem services [12,13] should also be considered as discussed below.

In order to inform policy on whether to promote the conservation, management, restoration or construction of wetlands to mitigate the impacts of N, P and SS from agriculture, it is imperative that policy makers are provided with accurate, robust and independently reviewed information. Whilst there is a great deal of published material on this subject, a rigorous, independent systematic review has not been conducted. The current UK guidelines do not stipulate a target percent reduction of nutrient or SS concentrations required from wetlands and simply state that any reduction is sufficient. However, the Environment Agency (EA) wish to adopt the approach that some other European countries now have in place (e.g. Denmark), where a target percentage reduction is specified for different catchments. Hence DEFRA has commissioned a systematic review on how effective and what influences the effectiveness of wetlands at mitigating $\mathrm{N}, \mathrm{P}$ and $\mathrm{SS}$ inputs from agriculture to receiving freshwater in England. It is also important to consider the potential trade-off between other ecosystem services perceived to be provided by wetlands, such as carbon sequestration and habitat provision $[12,13]$ during the development of any policy. This will be included as a secondary outcome within the review. The review will be impartial and transparent and will follow the guidance set out by the Collaboration for Environmental Evidence (CEE). To exclude bias, the review will encompass a comprehensive literature search on all available material on the subject, both published and unpublished within the British Isles. Specific inclusion criteria will be adhered to and a formal assessment of the quality and reliability of the studies will be undertaken. The data will then be extracted and a data synthesis undertaken. The systematic review will be used as a basis from which to review current, and guide future, policy. Stakeholders include DEFRA and the EA.

\section{Objective of the review Primary question}

How effective are reedbeds, ponds, restored and constructed wetlands at retaining nitrogen, phosphorus and suspended sediment from agricultural pollution in England? (Table 1).

\section{Secondary question}

What are the characteristics of the whole system that determine how effective an established reedbed/pond or restored or constructed wetland will be at reducing $\mathrm{N}, \mathrm{P}$ \& SS inputs from agricultural pollution in England?

\section{Review scoping}

A review scoping exercise was undertaken in order to guide the construction of this protocol. The question at the start of the review scoping exercise was: 'Does the presence of a wetland reduce agricultural pollutants in receiving freshwater?'

\section{Developing and testing a search strategy}

The initial review scoping question was used in the development of effective keywords for searching. ISI Web of Knowledge was used as the test database of peer reviewed literature. The first search string returned over 600,000 hits. In order to reduce this number, the search string was then developed using an iterative process, and refined by language (English) and countries/territories= ( $\mathrm{N}$ Ireland OR Ireland OR UK OR Wales OR England OR Scotland ). The search string chosen for progression onto the study inclusion stage returned 3870 hits. In order to test the search strategy, this list of articles was checked for inclusion of a selection of papers identified as relevant to the review question.

\section{Assessing the volume of literature}

The papers were sorted by publication date with 10 papers per page, and a $10 \%$ sample of the 3870 papers was taken by selecting the top paper from each page. This $10 \%$ was then filtered at title level, followed by abstract level and finally at full text level, using the following inclusion criteria:

- Relevant subjects: Any water quality agricultural pollutant from waste in Europe.

- Type of intervention: Any freshwater wetland.

- Types of comparator: Studies with the following comparators were included:

1. Input concentration/load of pollutant to wetland versus output concentration/load of pollutant from wetland;

2. Concentration/load of pollutant from agricultural pollution entering receiving water with no wetland versus with a wetland (provided input and geography are comparable);

3. Concentration/load of pollutant entering receiving water before installation of a wetland versus after installation of a wetland (provided input and geography are comparable).

4. Upstream concentration of pollutant in receiving water versus downstream concentration of 
Table 1 Definition of components of the primary systematic review question

\begin{tabular}{|c|c|c|c|}
\hline Subject (Population) & Intervention & Comparator & Outcome \\
\hline Water bodies receiving: & Wetland: & $\begin{array}{l}\text { - Input vs output concentration to } \\
\text { wetland }\end{array}$ & $\begin{array}{l}1^{\circ}: \text { Percentage change in water quality } \\
\text { measure }\end{array}$ \\
\hline - Nitrogen (N) & - Constructed/treatment & - No wetland vs with wetland present & $\begin{array}{l}2^{\circ} \text { : Change in other parameters, including } \\
\text { biodiversity measures and greenhouse gas } \\
\text { emission; are there any synergies/trade-offs, } \\
\text { e.g. flood risk? }\end{array}$ \\
\hline - Phosphorus (P) & - Restored & - Before wetland vs with wetland present & \\
\hline \multirow[t]{2}{*}{ - Suspended solids/sediment (SS) } & - Reedbeds & & \\
\hline & - Ponds & & \\
\hline
\end{tabular}

pollutant in receiving water was NOT included due to the possibility of in-stream processing/ additional inputs.

- Types of outcome: The primary outcome was any quantitative change in pollutant concentration or load.

- Types of study: Studies on both full scale wetlands, pilot scale wetlands and laboratory studies were included. Modelling studies were not included.

From the sample of 387 papers, 50 papers were identified at title level, 28 at abstract level and 24 at full text level. This scaled up to 240 papers from the ISI Web of Knowledge search (6\% of the articles retrieved). As only one electronic database was used in the scoping exercise, and the inclusion of other databases and websites in the full scoping exercise would increase this number, it was agreed given budget constraints that refinement of the review question and inclusion criteria was required.

A crude study quality assessment of the final 24 articles was conducted and these were ranked either high, medium or low. The number of articles reporting on each agricultural pollutant, wetland type and specific to agricultural waste (and combinations thereof) was also tallied. This information, alongside discussions with the key stakeholder groups (DEFRA and the EA), helped refine and formulate the review question and inclusion criteria laid out in this protocol.

\section{Data extraction and analysis}

A trial data extraction was conducted on 16 of the articles that met the inclusion criteria at full text level. This included 12 peer reviewed primary research articles, 3 review papers and one book chapter. Where possible, the following data was extracted from each paper:

Location (e.g.: SW England); Annual rainfall; Soil drainage; Hydroperiod; Hydrogeomorphic lanscape setting; Wetland type (e.g.: reedbed); Size; Hydraulic loading; Hydraulic retention time; Management; Input; Time established; Monitoring frequency; Monitoring time; Seasons monitored; Analytes; Percentage reduction.

Where percentage reduction was not directly reported, it was calculated from data supplied within the paper if possible. The quality and type of data retrieved from the articles was variable. A complete dataset of all the parameters listed was not provided in any of the articles. The three review papers contained data on a lower number of parameters than the papers containing primary data.

Within the 16 articles, data from 43 different wetland systems was extracted, comprising of 33 constructed wetland systems and 10 natural wetlands, with size and length of time established ranging from $0.788 \mathrm{~m}^{2}$ to $40000 \mathrm{~m}^{2}$ and 0 to 15 years respectively.

In order to formulate a data synthesis strategy, three data analysis experts were consulted. This included two statisticians, one of whom was experienced in systematic reviews. Based on the sample data extraction, there was a consensus that given the large number of parameters (covariates), and number of data gaps, a traditional meta-analysis of the data would be challenging and may not be possible.

\section{Methods}

\section{Search strategy}

The search aims to capture an unbiased and comprehensive sample of the literature relevant to the question, whether published or unpublished. Different sources of information will be searched in order to maximise the coverage of the search. Bibliographies of any review papers, summary reports or books retrieved, will be searched for relevant references.

\section{Electronic databases}

The following electronic databases will be searched:

1. ISI Web of Knowledge

2. Copac

3. Agricola

4. JSTOR 


\section{EThOS}

6. DART - Europe E-theses Portal

No restrictions will be applied regarding the year of publication. The search will be refined by language (English) and country (UK, England, Scotland, Wales, Ireland) where this facility is available.

\section{Conservation and statutory websites}

The official websites for the following organisations will be searched:

1. Department of Environment, Food and Rural Affairs (DEFRA)

2. Wildfowl and Wetlands Trust (WWT)

3. Ramsar

4. Environment Agency (EA)

5. English Nature

6. Countryside Council for Wales

7. Department of Agriculture and Rural Development (DARD)

8. Scottish Natural Heritage (SNH)

9. Water Framework Directive

10. Scottish Environment Protection Agency (SEPA)

11. Department of Agriculture, Food and the Marine

12. The Irish Agriculture and Food Development Agency (TEAGASC)

13. Constructed Wetland Association

14. Wetlands International

\section{Websites}

The following search engines will be searched:

1. www.google.com

2. scholar.google.co.uk

3. www.dogpile.com

The first 50 hits from each search will be examined for relevance, with any links present being followed only once from the original hit. Bibliographies of articles viewed at full text will be searched.

\section{Authors, recognised experts and practitioners}

Authors, recognised experts and practitioners (to include the society of wetland scientists) will also be contacted for further recommendations and for the provision of any unpublished material or missing data that may be relevant.

\section{Search terms}

Combinations of the below search terms (Table 2) will be used (where * denotes a wildcard term) to search the databases and websites. Search terms within each group will be combined using the Boolean OR operator, and between groups using the Boolean AND operator where possible.
Table 2 Search terms

\begin{tabular}{|c|c|c|}
\hline Group 1 & Group 2 & Group 3 \\
\hline Intervention elements & Population elements & Location elements \\
\hline - Wetland & - Nutrient & - UK \\
\hline - Pond & - $P$ & - United Kingdom \\
\hline - Marsh & - Phos* & - Brit* \\
\hline - Fen & $\bullet N$ & - Engl* \\
\hline - Floodplain & - Nitr* & - $\operatorname{Scot}^{*}$ \\
\hline - Bog & - $\mathrm{Amm}^{*}$ & - Wales \\
\hline - Mire & - Sediment & - Welsh \\
\hline - "Reed bed" & - Suspended solid & - Ireland \\
\hline - Reedbed & - Agricultural runoff & - Irish \\
\hline • "Riparian zone" & - Farm & \\
\hline \multicolumn{3}{|l|}{ - Oxbow } \\
\hline \multicolumn{3}{|l|}{ - "Riparian forest" } \\
\hline \multicolumn{3}{|l|}{ - Scrapes } \\
\hline - Berms & & \\
\hline
\end{tabular}

Search terms from group 3 will be used when the facility to refine the search by country is not available. Search terms will be tailored as necessary to the specific data base or search engine used, and as the search progresses.

\section{Study inclusion criteria}

Once the search has been conducted, inclusion criteria will be applied in order to identify relevant articles. The articles will be filtered at three levels; by title, then abstract (or introduction section if abstract is not available), and finally by full text. Citations will be stored in Endnote.

- Relevant subjects: Water bodies receiving N, P and SS from agricultural waste in England, lowland Wales and Ireland. All forms of $\mathrm{N}$ and P, and all types of agricultural waste will be included.

- Type of intervention: Freshwater constructed or restored wetlands, to include ponds, marshes, fens, floodplains, bogs, mires and reedbeds. Established reedbeds or ponds will also be included.

- Types of comparator: Studies with the following comparators will be included:

1. Input concentration/load of N, P, or SS to wetland versus output concentration/load of $\mathrm{N}, \mathrm{P}$ or SS from wetland;

2. Concentration/load of N, P, or SS from agricultural pollution entering receiving water with no wetland versus with a wetland (provided input and geography are comparable); 
3. Concentration/load of N, P, or SS entering receiving water before installation of a wetland versus after installation of a wetland (provided input and geography are comparable).

4. Upstream concentration of $\mathrm{N}, \mathrm{P}$ or $\mathrm{SS}$ in receiving water versus downstream concentration of $\mathrm{N}, \mathrm{P}$ or SS in receiving water will NOT be included due to the possibility of in-stream processing/ additional inputs.

- Types of outcome: The primary outcome is a quantitative change in N, P or SS concentration or load. Quantitative changes in different species of $\mathrm{N}$ (e.g. nitrate and ammonium) and $\mathrm{P}$ will be included. The secondary outcome is a change in other water quality parameters, biodiversity, or greenhouse gas production. The secondary outcome will not be used as an inclusion criterion.

- Types of study: Studies on both full scale wetlands and pilot scale wetlands will be included. Studies on laboratory mescosms and modelling studies will not be included.

Once the abstracts have been screened, the consistency of the above process will be checked. If in doubt about inclusion at abstract level, the whole text will be viewed. Two reviewers will assess a random subset of $10 \%$ of the original list of citations, applying the inclusion criteria at title and abstract level. The level of agreement between the two reviewers will be measured by kappa analysis, with a kappa rating of 0.6 or above ('substantial' agreement, compared to chance of agreement alone) considered acceptable. If kappa is less than 0.6, the discrepancies will be discussed and resolved by consensus. The reviewers will then clarify or modify the inclusion criteria as necessary, and one reviewer will then apply the inclusion criteria to the rest of the citations.

\section{Study quality assessment}

Articles which meet the inclusion criteria will be viewed at full text, and either be excluded or assigned different categories of study quality (poor, medium, high). At least two reviewers will independently assess a random subset of $25 \%$ articles viewed at full text. The level of agreement between the two reviewers will be measured by kappa analysis, with a kappa rating of 0.6 or above considered acceptable. If kappa is less than 0.6 , the discrepancies will be discussed and resolved by consensus. A possible ranking system is suggested below:

High: Established wetland ( $>5$ years); Regular monitoring (>=weekly); Long-term monitoring ( $>=1.5$ years); Full scale wetland; Scientifically rigorous (methodological and analytical); Good control.
Medium: Scientifically rigorous (methodological and analytical); Good control; Either full or pilot scale wetland; and one or more (but not all) of either; Established wetland; Regular monitoring ( $>=$ weekly); Long-term monitoring ( $>=1.5$ years);

Low: Poor control or lacking scientific rigour.

\section{Data extraction strategy}

The following data will be recorded on a specially designed data extraction form to include the following information where available:

General location; geology; soil characteristics; hydro geomorphic landscape setting; type of wetland; type of vegetation; area of wetland; type of management; hydroperiod; hydraulic loading; hydraulic retention time; size of the area generating the pollution; ratio of area generating waste to area of wetland; type of waste; agricultural intensity of upstream area; length of time wetland has been established; frequency of monitoring; length of time monitored; seasons monitored; analytes measured; control type; input and output (without/with; before/ after) concentrations/loads/populations of any analytes measured; reduction/increase/no change; \% reduction.

\section{Data synthesis}

A descriptive statistical overview of the data will be presented. For each analyte this will show: the number of sites, \% of sites showing a reduction, \% of sites showing an increase and a mean \% reduction with standard deviation, calculated from averaged values presented in the source studies.

If possible, a more rigorous statistical analysis of the data will be conducted to examine the effect of the covariates (effect modifiers) on the efficiency of a wetland's ability to remove (or otherwise) N, P or SS. These covariates include: Geology; soil characteristics; hydro geomorphic landscape setting; type of wetland; type of vegetation; area of wetland; type of management; hydroperiod; hydraulic loading; hydraulic retention time; size of the area generating the pollution; ratio of area generating waste to area of wetland; type of waste; agricultural intensity of upstream area; length of time wetland has been established; frequency of monitoring; length of time monitored; seasons monitored. How these covariates are categorised will depend on the quality and type of data retrieved during the data extraction stage. This will start with constructing a binomial generalised linear model with the possibility of constructing a Bayesian generalised linear model. External expertise will be made use of where necessary in order to formulate these models. In the event of none of the covariates showing a significant control on whether a wetland reduces or increases the analyte concentration using the extracted data, a narrative synthesis will be conducted. 


\section{Competing interest}

No conflicts of interest to be declared.

\section{Authors' contributions}

EPF wrote the protocol document and undertook the review scoping exercise. MA, JV, MS, EM, CS, and JN helped formulate the scientific scope of the document, provided expertise on wetlands and wetland processes, and reviewed and edited the protocol. JM provided expertise on the systematic review process and reviewed and edited the protocol. DC helped formulate the stakeholder element of the protocol and reviewed and edited the protocol. All authors read and approved the final manuscript.

\section{Authors' information}

Review Team: Elizabeth Palmer-Felgate (CEH), Mike Acreman (CEH), Jos Verhoeven (University of Utrecht), Miklas Scholz (The University of Salford), Edward Maltby (University of Liverpool), Charlie Stratford (CEH), Jonathan Newman (CEH), James Miller (CEH), Deborah Coughlin (DEFRA).

\section{Funding}

This systematic review is funded by the Department for Environment, Food and Rural Affairs (DEFRA).

\section{Author details}

${ }^{1}$ Centre for Ecology and Hydrology, CEH Wallingford, Maclean Building, Crowmarsh Gifford, Wallingford, Oxfordshire OX10 8BB, UK. ${ }^{2}$ Ecology and Biodiversity, Universiteit Utrecht, H.R. Kruytgebouw, N310 Padualaan 8, 3584 $\mathrm{CH}$, Utrecht, The Netherlands. ${ }^{3}$ Civil Engineering Research Centre, School of Computing, Science \& Engineering, The University of Salford, Salford, Greater Manchester M5 4WT, UK. ${ }^{4}$ Emeritus Professor, Wetland Science, Water \& Ecosystem Management, University of Liverpool, Liverpool L69 3BX, UK. ${ }^{5}$ Analysis \& Evidence Team, Water \& Flood Risk Management, Defra, Area 2B, Ergon House, c/o Nobel House, 17 Smith Square, London SW1P 3JR, UK.

Received: 4 October 2012 Accepted: 23 January 2013

Published: 29 January 2013

\section{References}

1. EU: European Parliament and the Council of the European Union: Directive 2000/60/EC. Establishing a framework for community action in the field of water policy. 2000:152.

2. Heathwaite AL, Johnes PJ, Peters NE: Trends in nutrients. Hydrological Processes 1996, 10(2):263-293.

3. Edwards AC, Withers PJA: Transport and delivery of suspended solids, nitrogen and phosphorus from various sources to freshwaters in the UK. Journal of Hydrology 2008, 350(3-4):144-153.

4. Schwartz JS, Simon A, Klimetz L: Use of fish functional traits to associate in-stream suspended sediment transport metrics with biological impairment. Environmental Monitoring and Assessment 2011, 179(1-4):347-369.

5. Palmer-Felgate EJ, Jarvie HP, Withers PJA, Mortimer RJG, Krom MD: Streambed phosphorus in paired catchments with different agricultural land use intensity. Agriculture Ecosystems \& Environment 2009, 134(1-2):53-66.

6. Fisher J, Acreman MC: Wetland nutrient removal: a review of the evidence. Hydrol Earth Syst Sc 2004, 8(4):673-685.

7. Braskerud BC, Tonderski KS, Wedding B, Bakke R, Blankenberg AGB, Ulen B, Koskiaho J: Can constructed wetlands reduce the diffuse phosphorus loads to eutrophic water in cold temperate regions? J Environ Qual 2005, 34(6):2145-2155.

8. Verhoeven JTA, Arheimer B, Yin C, Hefting MM: Regional and global concerns over wetlands and water quality. Trends in Ecology \& Evolution 2006, 21(2):96-103.

9. Stratford CJ, Duenas M, Bowes M, Palmer-Felgate EJ, Mackenzie S: 10 years on: How the nutrient removal performance of a treatment reed bed changes with time. In 2nd Irish Internation Conference on Constructed Wetlands for Wastewater Treatment and Environmental Pollution Control. Conference Proceedings Dublin, Ireland; 2010.

10. Palmer-Felgate EJ, Mortimer RJG, Krom MD, Jarvie HP, Williams RJ, Spraggs RE, Stratford CJ: Internal loading of phosphorus in a sedimentation pond of a treatment wetland: Effect of a phytoplankton crash. Science of the Total Environment 2011, 409(11):2222-2232.
11. Scholz M, Harrington R, Carroll P, Mustafa A: Monitoring of nutrient removal within integrated constructed wetlands (ICW). Desalination 2010, 250(1):356-360.

12. Acreman MC, Harding RJ, Lloyd C, McNamara NP, Mountford JO, Mould DJ, Purse BV, Heard MS, Stratford CJ, Dury SJ: Trade-off in ecosystem services of the Somerset Levels and Moors wetlands. Hydrological Sciences JournalJournal Des Sciences Hydrologiques 2011, 56(8):1543-1565.

13. Harrington R, Carroll P, Cook S, Harrington C, Scholz M, Mclnnes RJ: Integrated constructed wetlands: water management as a land-use issue, implementing the 'Ecosystem Approach'. Water Sci Technol 2011, 63(12):2929-2937.

doi:10.1186/2047-2382-2-1

Cite this article as: Palmer-Felgate et al.: How effective are reedbeds, ponds, restored and constructed wetlands at retaining nitrogen, phosphorus and suspended sediment from agricultural pollution in England?. Environmental Evidence 2013 2:1.

\section{Submit your next manuscript to BioMed Central and take full advantage of:}

- Convenient online submission

- Thorough peer review

- No space constraints or color figure charges

- Immediate publication on acceptance

- Inclusion in PubMed, CAS, Scopus and Google Scholar

- Research which is freely available for redistribution

Submit your manuscript at www.biomedcentral.com/submit
C) Biomed Central 